This paper has been published with the following reference

Bernabeu, M.; Moreno, M.; Llinares, S. (2021). Primary school students' understanding of polygons and the relationships between polygons. Educational Studies in Mathematics, 106, 251-270

https://doi.org/10.1007/s10649-020-10012-1

\title{
PRIMARY SCHOOL STUDENTS' UNDERSTANDING OF POLYGONS AND THE RELATIONSHIPS BETWEEN POLYGONS
}

\author{
Melanina Bernabeu ${ }^{1}$; Mar Moreno²; Salvador Llinares ${ }^{3}$ \\ Department of Innovation and Didactic Training, University of Alicante, Spain \\ ํㅡㄹ.lania.bernabeu@ua.es; ${ }^{2}$ mmoreno@ua.es; ${ }^{3}$ sllinares@ua.es \\ ${ }^{1}$ 0000-0001-7121-5795; ${ }^{20000-0002-6687-091 X ; ~}{ }^{3} 0000-0002-0801-316 \mathrm{X}$
}

\section{Acknowledgements}

This research was supported in part by the project Prometheus/2017/135 of the Generalitat Valenciana (Spain) and by the University of Alicante (FPU2017-014).

\begin{abstract}
The goal of this study was to characterise how third-grade primary school students understand the concept of polygon as well as relationships between polygons. Following a teaching experiment, students answered a questionnaire on the recognition of polygons and relationships between polygons, providing information about how a polygon was understood as an example of a class. Dimensional deconstruction of shapes (Duval, 2017) and Statistical Implicative Analysis (Gras et al. 2008) were used to analyse students' answers. The findings indicated that students' understanding of the polygon concept depended on how students recognised and modified the relevant attributes considered in the definition of polygon. These results suggest that a progressive complexity underlies the understanding of the concept of polygon. Evidence of this progressive complexity was found in the relationship between recognising a figure as a polygon and transforming a non-example of a polygon into a polygon. Furthermore, the ability to identify a polygon as an instance of a class depended on the attribute that defines the class. That is, the fact of identifying a class of polygons was linked to the non-relevant attribute considered. Instructional implications are finally drawn regarding the key role of dimensional deconstruction to understand polygons and relationships between polygons.
\end{abstract}

Keywords: Concept of polygon; Dimensional deconstruction; Geometric thinking; Implicative Analysis; Primary education. 
Understanding a polygon as an example of a class represents a step forward in the geometric thinking of primary school students (Battista 2007; Clements and Battista 1992; Sinclair et al. 2017; Yesil-Dagli and Halat 2016). Some studies indicate that when students are learning to recognise a closed plane figure with straight and noncrossed sides as a polygon, they inductively create perceptual images that are often difficult to replace. These perceptual images make it difficult to consider a certain polygon as an example of a class, since the perceptual images are linked to the typicality of the representations used (Clements et al. 1999; Hershkowitz 1990; Mesquita 1998; Tsamir et al. 2008). An illustration of this is when a square (a quadrilateral with congruent sides and angles) represented with its sides parallel to the edge of the sheet is presented as an example of a quadrilateral, or when only an equilateral triangle (a triangle with three equal sides) is shown as an example of the concept of triangle (Tsamir et al. 2008). Hershkowitz (1989) indicated that students sometimes impose conditions derived from prototypical examples to determine whether a polygon is an example of a class. The definition of a geometrical concept is regarded as a criterion for classifying examples and non-examples of this concept. A non-example of a concept is when the figure does not fulfil some relevant attribute of the concept's definition. Furthermore, learning to identify a figure's additional attributes is the first step towards classifying and recognising a figure as an instance of a class (Hershkowitz 1990). Such learning has been shown to be problematic due to the difficulties in recognising the attributes of different figures. The latter was demonstrated in the case of defining and classifying quadrilaterals in secondary school (Fujita and Jones 2007; Fujita 2012; Fujita et al. 2019) and in the case of kindergarten children's grasp of non-examples of triangles (Tsamir et al. 2008). Identifying non-critical attributes of polygons to consider a class of polygons requires suitable interactions between concepts and images.

Fischbein (1993) established the idea of figural concept to underline the symbiosis between figure and concept i.e. the relationship between the perceptive — such as the recognition of certain attributes-and the logical conditions governing the concept—such as the mathematical properties that define a class. Two illustrations of this would be secondary students' cognitive abilities to code and decode representations of 3D shapes (Pittalis and Christou 2013) and kindergarten students recognising examples and non-examples of specific polygons (Halat and Yesil-Dagli 2016; Tsamir et al. 2008). The relationship between figure and concept is also visible when trying to classify figures because students need to manipulate the figures mentally to identify similarities and differences. Duval (2017) underlined this relationship when linking the understanding of geometrical figures to the coordination of two semiotic representation systems: the discursive (oral or written) and the non-discursive (drawings, sketches, geometric figures) and making mathematical sense of parts of figures when students are reasoning about figures. This theoretical approach allows us to elucidate sense-making processes involving multiple representations (Fujita et al. 2019). One example is when symmetry is identified as the attribute that defines a group of symmetric polygons in item involving drawing and verbal explanations. This characterisation stresses how important it is that the perceptual recognition of the geometric figure be controlled by geometric properties (definition, theorems, properties, etc.) in order to infer that a figure is an instance of the class of symmetric figures as established by Fischbein (1993).

One aspect in this geometric thinking development is linked to understanding inclusive relationships between classes of polygons. For example, when considering that a square can be an example of a rhombus (quadrilateral with four equal sides). The classification process depends on the ability to identify similarities and differences between figures and to explain why a figure is an example of a class (Walcott et al. 2009). Evidence that students understand inclusive relationships between figures is found when students are able to distinguish figures according to their properties (attributes) and not only according to their perceptual appearance (Battista 2007; Clements and Battista 1992). It thus seems logical that the ability to understand inclusive relationships is linked to the ability to identify examples and non-examples of types of polygons (De Villiers 1994; Tsamir et al. 2015). For example, if we define a trapezoid in a partitive way as a quadrilateral with only a pair of parallel sides, parallelograms (quadrilaterals with opposite parallel sides) would be excluded from that class. But if we define a trapezoid inclusively as quadrilaterals with at least one pair of parallel sides, parallelograms would represent a specific case of trapezoid.

From this perspective, accepting a figure as an example of a class of polygons complements students' understanding of geometric figures. Previous research has characterised the understanding of inclusive classifications in pre-school students (Halat and Yesil-Dagli 2016; Kaur 2015) and in secondary students (Fujita et al. 2019; Monaghan 2000), as well as in pre-service teachers (Fujita 2012; Fujita \& Jones 2007; Kozakli-Ulger 
and Tapan-Broutin 2017; Wang and Kinzel 2014). Less attention has been given, however, to this phenomenon in primary education (Moreno et al. 2018; Sinclair et al. 2016). In addition, previous research had found that difficulties in classifying polygons were linked to the use of prototypical images to understand polygons and to the classification process itself (Bernabeu and Llinares 2017; Bernabeu et al. 2018; De Villiers 1994; Kaur 2015).

The research presented here aims at building knowledge about how third-grade primary students understand that a polygon can be considered as an example of a class of polygons. In other words, the aim was to understand how third-grade primary school students identify polygon attributes and use them to determine whether a polygon belongs to a class.

\section{THEORETICAL FRAMEWORK}

Duval $(1995,1999)$ emphasised the importance of coordinating perceptual, discursive, sequential and operational apprehensions and how students make mathematical sense of parts of figures to understand geometrical figures. Perceptual apprehension refers to recognising and naming a figure based on a global perception and it determines one's ability to recognise sub-configurations. Sequential apprehension is mobilised when a figure is constructed or its construction is described - when drawing a figure that fulfils certain conditions for example. Discursive apprehension consists in associating the figure with mathematical properties. Finally, operational apprehension allows modifying a figure during the problem-solving process. From this perspective, understanding the polygon concept is based on the relationship between the following processes: recognising a figure's attributes to establish whether or not it is a polygon; associating the figure with its mathematical properties (discursive apprehension); and being able to draw polygons that meet certain conditions or to modify a figure so that it becomes a polygon (sequential apprehension). Furthermore, reasoning based on the figure's attributes is understood as the ability to justify why a figure is an example of a class. That is, to be able to justify that two perceptually different figures can be considered as examples of the same class.

To analyse a figure, from a mathematical viewpoint, a student needs to identify sub-configurations in the figures, which Duval (2017) calls dimensional deconstruction. Dimensional deconstruction allows to identify and compare parts of figures. For example, considering the length of a triangle's sides to determine the type of triangle when triangles are classified according to the length of their sides. According to Duval, dimensional deconstruction implies a cognitive leap for students as it permits them to see the figures mathematically. According to Fischbein (1993), this is when mathematical properties begin to govern the perceptual approach. For example, identifying an internal angle above $180^{\circ}$ in a figure as a common attribute to a group of figures is more challenging than simply recognising this attribute in one figure. In addition, classifying figures implies identifying an attribute that is common to different figures, and determining that other figures that do not have this attribute do not belong to this class. For Duval (2017), this is a mathematical approach to figures that goes beyond the perceptual recognition of similarities and differences illustrating the relationship between the perceptual approach and mathematical properties that define the concept (Fischbein 1993).

Developing dimensional deconstruction implies recognising a figure's attributes and identifying what is common to different figures. This latter ability allows considering the different figures as examples of the same class from a mathematical viewpoint. Although both processes of recognising and identifying consist in making specific attributes explicit rather than recognising the figures globally, identifying mathematical aspects common to different figures is more challenging. According to Duval (2017), we refer to a figure's properties using words that usually imply a dimensional deconstruction with respect to what the figure allows us to see. We then use the properties stated in the definitions to deduce and perceive something else. Thus, the dimensional deconstruction involved in the process of recognising attributes and identifying similarities between figures may indicate how students come to understand the classification of geometric figures. The coordination of dimensional deconstruction and cognitive apprehensions lead us to examine four processes that underline the understanding of polygons: recognising examples and non-examples of polygons; identifying an attribute common to a set of polygons; determining whether a polygon belongs to a class; and drawing polygons according to certain conditions. These processes are similar to the geometrical ability to represent 3D shapes, understood as the two representational/cognitive abilities of primary and secondary school students to decode and code plane representations of 3D shapes (Pittalis and Christou 2013). In this latter case, we hypothesise that decoding 
consists in the ability to recognise the structural elements of 3D figures (vertices, faces and edges) according to various representations modes and to interpret geometrical properties in plane representations of 3D shapes (Pittalis and Christou 2013).

Based on these theoretical premises, we defined the research question below.

How do third grade primary students reasoning with the attributes of a polygon to consider it as an example of a class?

\section{METHOD}

\section{Participants and curricular context}

The participants in this study were 59 students ( 37 boys and 22 girls) in their third year of primary education (aged 9 to 10 years), in two different classes in a school in Spain. One of the researchers was the teacher in both classes. The students had not received any previous instruction regarding the concept of polygon. The Spanish mathematics curriculum in third grade contains activities where pupils have to recognise and name different polygons according to their number of sides, concavity and symmetry. Less attention is given, however, to considering relationships between types of figures. In previous courses, teachers used individual work sheets and textbooks that included tasks where students had to recognise, draw and name prototypical figures such as the square, rectangle, triangle or circle. The third year was selected in order to focus on developing the polygon concept and on identifying classes of polygons.

\section{Teaching experiment}

The teaching experiment aimed at encouraging dimensional deconstruction by coordinating cognitive apprehensions (Duval 1995, 2017). To do this, the tasks were directed towards recognising examples and nonexamples of polygons as well as recognising different attributes in the polygons, such as the number of sides, concavity (at least an internal angle higher than $180^{\circ}$ ), axes of symmetry and parallelism in the quadrilaterals. We also presented tasks in which one had to identify when a polygon was an example or a non-example of a class of polygons and consider types of triangles according to their sides (equilateral: triangle with three equal sides; isosceles: triangle with at least two equal sides; and scalene: triangle with any equal side) and/or angles (acute triangle: triangle with three acute angles; right triangle: triangle with a right angle; and obtuse triangle: triangle with one obtuse angle) as well as types of quadrilaterals according to the parallelism of their sides. In addition, students had to determine whether a figure belonged to a class and draw examples and non-examples of the class. In this teaching experiment, we used inclusive definitions. We indicated, for example, that an isosceles triangle has at least two equal sides, so equilateral triangles are a particular case of isosceles triangles; or that a trapezoid has at least two parallel sides, so parallelograms are a particular case of trapezoid. However, in the teaching experiment, we did not use the terms "exactly" or "at least" in the task descriptions to enable us to verify what kind of reasoning students were using. The teaching experiment consisted of 10 fifty-minute sessions (two sessions per week) over 5 weeks. All sessions shared the same methodological structure: introducing a task and resolving the task in a large group in which students explained and justified their answers and repeat this process with other tasks. The sessions ended with individual student-solving tasks.

\section{Instrument}

The students answered a questionnaire at the end of the teaching experiment composed of 6 items some of which were composed of several sections. The objective of these items was to allow inferring information about how students used apprehensions and dimensional deconstruction to give a mathematical sense to the parts of geometrical figures. The items were grouped into four dimensions:

\section{- Dimension 1 - Recognise polygons, (items 1a, 1b, 1c, 1d and 1e)}

Item 1a asked to recognise polygons among 15 geometric figures (8 polygons and 7 non-examples of polygons). Items 1b, 1c, 1d and 1e asked the students: to recognise which attributes had to be modified in a non-example of a polygon to turn it into a polygon; to explain this transformation; and to draw the polygon. The non-examples of polygon were figures that did not fulfil any attribute condition included in the definition: an open 2D figure with curved and intersecting sides (1b); a 2D open figure with a curved side (1c) (Figure 1); a closed 2D figure with intersecting sides and a curved side (1d); and an open 2D figure with intersecting sides (1e). 


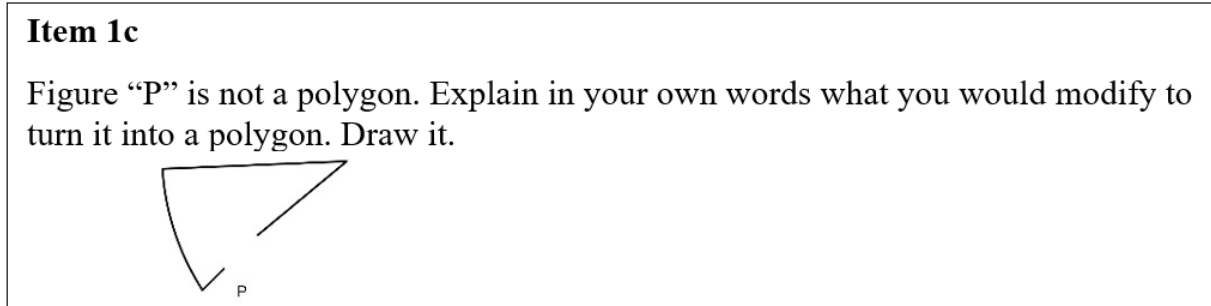

Figure 1. Item 1c: open figure with curved side

- $\quad$ Dimension 2 - Identify the common attribute in a set of polygons (items 2, 3, and 4a)

Items 2, 3 and 4a presented two groups of polygons. One group consisted of polygons that had a common attribute, and another group that did not have that attribute. The items asked to identify the common attribute, explain the relationship of belonging and to draw an example of a polygon belonging to that group and another polygon that did not belong to that group (a non-example). The attributes used were concavity (polygons with at least one internal angle over $180^{\circ}$ ) (item 2, Figure 2), polygons with six sides (item 3), and polygons with an axis of symmetry (item 4a). In items 2, 3, 4, 5 and 6 we used the metaphor of the Drawing Machine (MD), introduced by Battista (2012), which drew geometric figures under some conditions (examples) and could not make figures that did not meet these conditions (non-examples).

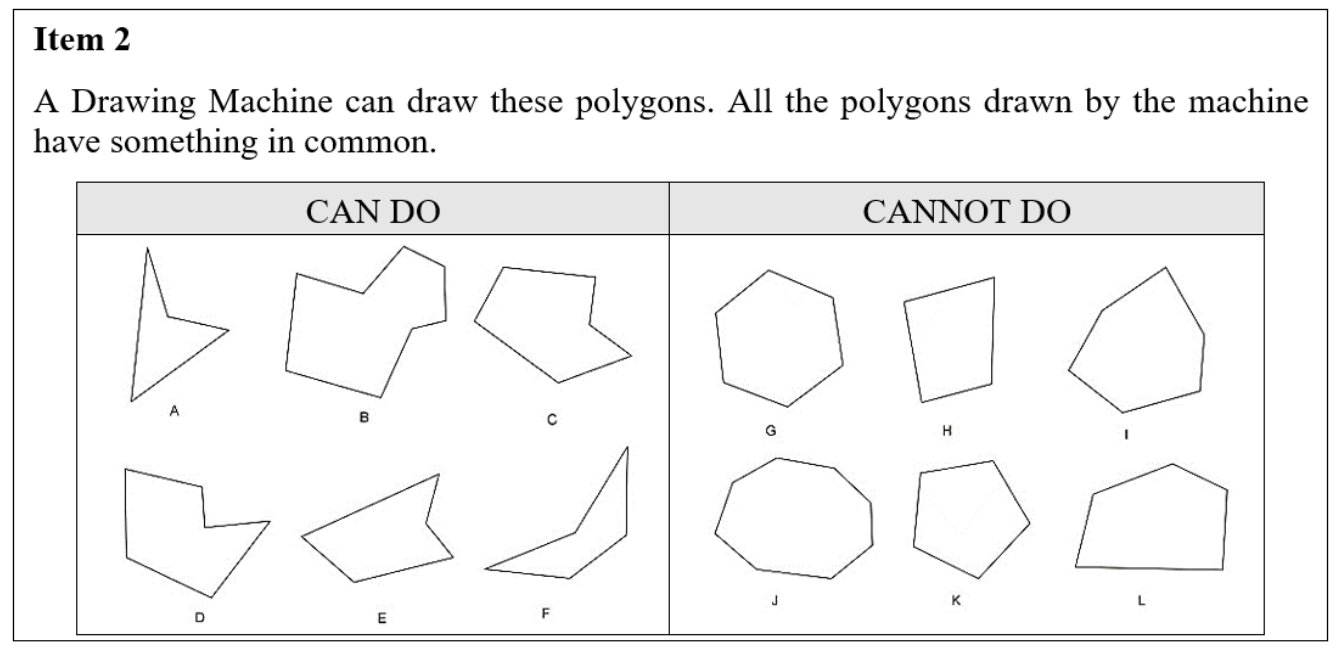

Figure 2. Item 2: concavity/convexity (adapted from Battista 2012)

- $\quad$ Dimension 3 - Determine whether a polygon belongs to a class (items 4b, 5 and 6b)

To classify polygons inclusively, students had to identify the common attribute of a group of polygons (item 4a and 5) or the attribute inferred from a discursive description (item 6b) and then determine whether a polygon belonged to the class or not. For example, considering a rhombus (a figure with two axes of symmetry) as an example of the class of polygons with an axis of symmetry (item 4b); an equilateral triangle (a triangle with three equal sides) as an example of the class of isosceles triangle (a triangle with at least two equal sides) (item 5, Figure 3); and a parallelogram (a quadrilateral where opposite sides are parallel) as an example of a trapezoid (a quadrilateral with at least one pair of parallel opposite sides) (item 6b, Figure 4). 


\section{Item 5}

A Drawing Machine can draw these polygons. All the polygons drawn by the machine have something in common.

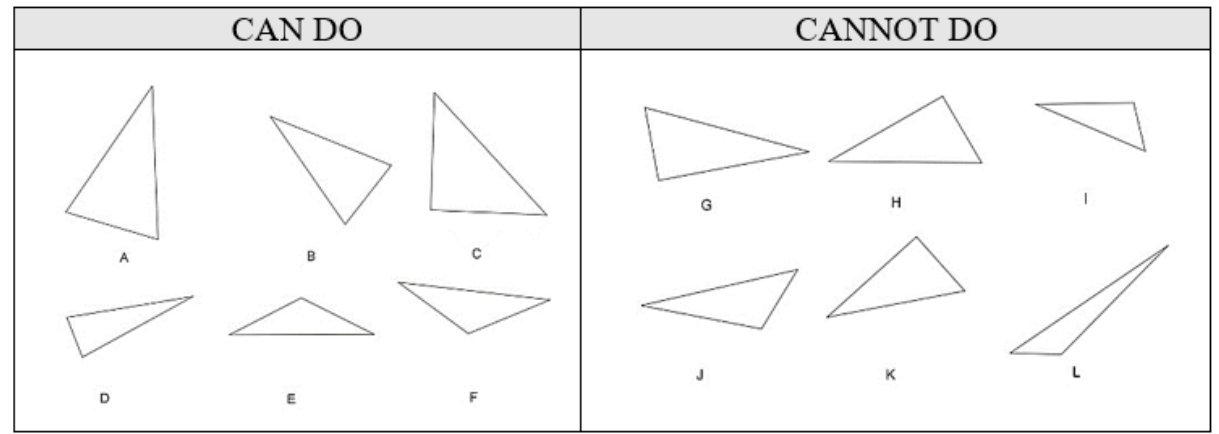

a) Can the Drawing Machine draw figure M? Why?

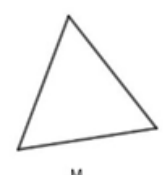

Figure 3. Item 5: Considering an equilateral triangle as an example of an isosceles triangle

\section{Item $6 \mathrm{~b}$}

A Drawing Machine can draw Quadrilaterals (four-sided polygons) with two parallel sides. Can the Drawing Machine draw the figure "A"? Why?

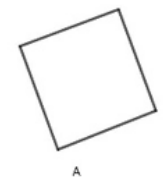

Figure 4. Item 6b: Considering a square as an example of a quadrilateral with parallel sides

- $\quad$ Dimension 4 - Draw polygons with determined attributes (item 6a).

Item 6a asks to draw three quadrilaterals with two parallel sides and three quadrilaterals that do not fulfil this condition (Figure 5).

\section{Item 6}

A Drawing Machine can draw:

Quadrilaterals (polygons with four sides) with two parallel sides

a) Draw three quadrilaterals that the machine can draw, and three quadrilaterals the machine cannot draw

\begin{tabular}{|l|l|}
\hline \multicolumn{1}{|c|}{$\begin{array}{c}\text { CAN DO } \\
\text { Quadrilaterals with two parallel sides }\end{array}$} & CANNOT DO \\
\hline 1. & 1. \\
\hline 2. & 2. \\
\hline 3. & 3. \\
\hline
\end{tabular}

Figure 5. Item 6a: drawing polygons that fulfil certain conditions 


\section{Analysis}

To analyse the students' answers, we generated 48 variables from the four dimensions of the questionnaire:

- $\quad$ D1- Recognise polygons $(\mathrm{n}=27)$;

- $\quad$ D2- Identify the common attribute in a group of polygons $(n=15)$;

- $\quad$ D3- Determine whether a polygon belongs to a class $(\mathrm{n}=3)$; and

- $\quad$ D4- Draw polygons with given attributes $(\mathrm{n}=3)$ (Table 2).

For the item recognise polygons (item 1a) amid 15 figures, we assigned a number to each figure, generating a total of 15 variables (variables from 1a.1 to 1a.15). The items transform non-examples of polygons into polygons (items 1b, 1c, 1d and 1e) showed figures that did not fulfil some of the attributes in the polygon's definition. For each figure, we considered three variables: explain, draw and one variable for explaining and drawing together. Since there were four figures, we generated a total of $n=12$ variables. Table 1 shows the structure of the variables of Dimension 1.

Table 1. Variables for the analysis of Dimension 1: Recognise polygons

\begin{tabular}{|c|c|c|c|c|c|}
\hline DIMENSION 1 & ITEM & \multicolumn{4}{|c|}{ GEOMETRIC FIGURES } \\
\hline Recognise polygons & $1 \mathrm{a}$ & \multicolumn{4}{|c|}{ 1a.1 ... 1a.15 (8 polygons and 7 non-examples of polygons) } \\
\hline & & ATTRIBUTES & EXPLAIN & DRAW & EXPLAIN AND DRAW \\
\hline \multirow[t]{4}{*}{$\begin{array}{lc}\text { Turn a } & \text { non-example } \\
\text { polygon } & \text { into a } \\
\text { polygon } & \end{array}$} & $1 \mathrm{~b}$ & $\begin{array}{l}\text { Open 2D figure }+ \\
\text { curved side }+ \\
\text { intersecting sides }\end{array}$ & 1b.oci-e & 1b.oci-d & 1b.oci-e\&d \\
\hline & 1c & $\begin{array}{l}\text { Open 2D figure + } \\
\text { curved side }\end{array}$ & 1c.oc-e & 1c.oc-d & 1c.oc-e\&d \\
\hline & $1 \mathrm{~d}$ & $\begin{array}{l}\text { 2D figure with } \\
\text { curved side } \\
\text { intersecting sides }\end{array}$ & 1d.ci-e & 1d.ci-d & 1d.ci-e\&d \\
\hline & 1e & $\begin{array}{l}\text { Open 2D figure }+ \\
\text { intersecting sides }\end{array}$ & 1e.oi-e & 1e.oi-d & 1e.oi-e\&d \\
\hline
\end{tabular}

In the case of the three items related to identifying the common attribute in a set of polygons (items 2- concavity; item 3- six sides, and item 4a- symmetry), we generated 5 variables for each item (a total of 15 variables). Table 2 presents how we considered the variables explain and draw in the case of concavity. Table 3 shows the different variables related to the 3 items.

Table 2. Variables for analysis Dimension 2: Identifying the common attribute: concave/convex polygons

\begin{tabular}{|l|l|l|}
\hline $\begin{array}{l}\text { 2.cv/cx-e\&d: Explain and draw one } \\
\text { example (and one non-example) of } \\
\text { figure belonging to the group of } \\
\text { polygons showed (concave/convex } \\
\text { polygons) }\end{array}$ & EXPLAIN (e) & DRAW (d) \\
\hline CONCAVE (cv) & $\begin{array}{l}\text { 2.cv-e: explain the common } \\
\text { attribute in the concave polygon } \\
\text { group concave polygon (example of the } \\
\text { class) }\end{array}$ \\
\hline CONVEX (cx) & $\begin{array}{l}\text { 2.cx-e: explain the common } \\
\text { attribute in the convex polygon } \\
\text { group }\end{array}$ & $\begin{array}{l}\text { 2.cx-d: draw an example of a } \\
\text { convex polygon (example of the } \\
\text { class) }\end{array}$ \\
\hline
\end{tabular}


Table 3. Variables for the analysis of Dimension 2: Identify the common attribute in a group of polygons

\begin{tabular}{|c|c|c|c|c|c|}
\hline DIMENSION 2 & ITEM & ATTRIBUTE & EXPLAIN & DRAW & EXPLAIN AND DRAW \\
\hline \multirow{6}{*}{$\begin{array}{l}\text { Identify a common } \\
\text { attribute in a set of } \\
\text { polygons }\end{array}$} & \multirow[t]{2}{*}{2} & Concave (cv) & 2.cv-e & 2.cv-d & \multirow[t]{2}{*}{ 2.cv/cx-e\&d } \\
\hline & & Convex (cx) & 2.cx-e & 2.cX-d & \\
\hline & \multirow[t]{2}{*}{3} & Having 6 sides (6) & 3.6-e & 3.6-d & \multirow[t]{2}{*}{ 3.6/n6-e\&d } \\
\hline & & Not having 6 sides (n6) & 3.n6-e & 3.n6-d & \\
\hline & \multirow[t]{2}{*}{$4 a$} & Symmetry (s) & 4a.s-e & 4a.s-d & \multirow[t]{2}{*}{ 4a.s/ns-e\&d } \\
\hline & & No symmetry (ns) & 4a.ns-e & 4a.ns-d & \\
\hline
\end{tabular}

In the case of the items related to determining whether a polygon belonged to a class, we generated three variables. Variable 4b.sym-bel (belongs to symmetric polygons), a rhombus representing a polygon with an axis of symmetry (item 4b); variable 5.tri-bel (belongs to isosceles triangle), an equilateral triangle representing an isosceles triangle (item 5); and variable 6b.tzo-bel (belongs to quadrilaterals with two parallel sides), a square representing a quadrilateral with two parallel sides (item 6b). Table 4 illustrates this structure.

Table 4. Dimension 3 structure: variables for analysis

\begin{tabular}{|l|l|l|}
\hline DIMENSION 3 & ITEM & DESCRIPTION \\
\hline $\begin{array}{l}\text { Determine belonging } \\
\text { to a class. }\end{array}$ & $4 \mathrm{~b}$ & $\begin{array}{l}\text { 4b.sym-bel: Explain that a rhombus can be considered a polygon with an axis } \\
\text { of symmetry }\end{array}$ \\
\cline { 2 - 3 } & 5 & $\begin{array}{l}\text { 5.tri-bel: Explain that an equilateral triangle can be considered a triangle with } \\
\text { two equal sides }\end{array}$ \\
\cline { 2 - 3 } & $6 \mathrm{~b}$ & $\begin{array}{l}\text { 6b.tzo-bel: Explain that a square can be considered a quadrilateral with two } \\
\text { parallel sides (trapezoid) }\end{array}$ \\
\hline
\end{tabular}

To draw polygons with determined attributes based on the verbal description, we generated three variables (item 6a): draw at least two quadrilaterals with two parallel sides (examples) and at least two quadrilaterals without parallel sides (non-examples) (6a.e\&ne-d); draw at least two quadrilaterals with two parallel sides (6a.e-d); and draw at least two quadrilaterals without parallel sides (6a.ne-d). Table 5 presents the description of the variables considered in dimension 4: Draw polygons with determined attributes based on an oral description.

Table 5. Dimension 4: variables for analysis

\begin{tabular}{|l|l|l|}
\hline DIMENSION 4 & ITEM & DESCRIPTION \\
\hline $\begin{array}{l}\text { Draw polygons with } \\
\text { determined attributes } \\
\text { from an oral } \\
\text { description }\end{array}$ & $6 a$ & $\begin{array}{l}\text { 6a.tzo\&ntzo-d: Draw at least two quadrilaterals with two parallel sides } \\
\text { (trapezoid) and at least two quadrilaterals with no parallel sides (trapezium, } \\
\text { namely, non-trapezoid) }\end{array}$ \\
\cline { 2 - 3 } & 6a-1 & 6a.tzo-d: Draw at least two quadrilaterals with two parallel sides (trapezoid) \\
\cline { 2 - 3 } & 6a-2 & $\begin{array}{l}\text { 6a.ntzo-d: Draw at least two quadrilaterals without parallel sides (non- } \\
\text { trapezoid) }\end{array}$ \\
\hline
\end{tabular}

We assigned a value of 1 to each variable if the answer was correct and 0 if the answer was incorrect. We performed an implicative analysis (Gagatsis et al. 2006; Gras et al. 2008; Michael et al. 2006) to identify and organise quasi-logical implications (implicative relationships between the variables with a certain probability) using the C.H.I.C. (Classification Hierarchique, Implicative et Cohesitive), version 6.0. (Couturier 2008). We used implicative analysis because we wished to examine the nature of the dimensional deconstruction through the coordination of cognitive apprehensions i.e. when students recognise polygons, identify a common attribute in a set of polygons allowing to define a class and determine whether or not an instance of polygon belongs to the class. An implicative analysis assumes that knowledge is formed inductively (from the examples found) allowing students to ensure a certain extent of reliance on a rule (the property that determines a concept). Evidence of the rule is given by the way in which students answer an item, taking into account when students do not behave as expected (Trigueros and Escandón 2008). The quasi-implication between the variables- $\mathrm{Z} \rightarrow \mathrm{V}-$ indicates that if students answer $\mathrm{Z}$ affirmatively, then it is likely they answer $\mathrm{V}$ (though a relatively small number of answers may contradict this). That is, $\mathrm{Z} \rightarrow \mathrm{V}$ is equivalent to the fact that the set $\mathrm{Z} \wedge$ no $\mathrm{V}$ is almost null (in the sense that the set of observations $\mathrm{Z}$ is almost contained in V). In this paper, we use the arrow $\rightarrow$ to indicate a quasi-implication according to the meaning previously described.

This present analysis allows to illustrate students' knowledge of geometric facts, for example when they recognise that a polygon has a certain attribute and is therefore an example of a certain class of polygons. That 
is, when students are able to carry out dimensional deconstruction by coordinating the apprehensions to recognise parts of a figure presented in different representations. The implicative analysis takes into account students' answers that do not present this relationship. For example, the quasi-implication 1b.oci-e\&d $\rightarrow 1$ d.ci$e \& d$ indicates that if students are able to explain the transformation necessary to convert an open 2D figure with a curved side and intersecting sides into a polygon (1b.oci-e\&d) and draw the new polygon, then it is probable that they can explain and draw the transformation of a closed 2D figure with a curved side and intersecting sides into a polygon (1d.ci-e\&d). In addition, it indicates that the number of cases in which this implication is not met is relatively small (in fact it is almost nil, statistically).

Although quasi-implicative relationships are not transitive (Gras and Kuntz 2008), it is possible to interpret quasi-implications of the type $(\mathrm{V} \rightarrow \mathrm{Z}) \rightarrow \mathrm{N}$ to describe how students reason with respect to figure attributes. These quasi-implications are interpreted in the following way: if $(V$ and $Z$ ) then $\mathrm{N}$. For example, the quasiimplication 1d.ci-e\&d $\rightarrow$ 1c.oc-e\&d $\rightarrow 1 a 12$ (Figure 6) indicates that if students "turn an open figure with a curved side into a polygon and explain it" (1d.ci-e\&d) and "draw a polygon transforming an open 2D figure with a curved side explaining the transformation" (1c.oc-e\&d) then it is likely that they "recognise that a 2D figure with a curved side is not a polygon" (1a12). In addition, cases in which this quasi-implication is not fulfilled are statistically non-significant.

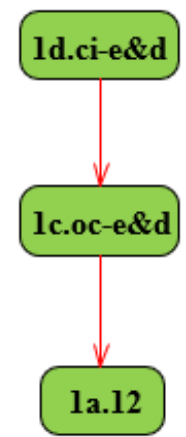

Figure 6. Example of implicative graph showing the relationships between the variables 1d.ci-e\&d, 1c.oc-e\&d and $1 a .12$

In this way, the implicative analysis allows us to assume certain quasi-implications between the variables although a relatively small number of answers may contradict it. This analysis provides information on the role played by different attributes in students' understanding of possible relationships between polygons.

\section{RESULTS}

The implicative graph, with a significance of $98 \%$ (Figure 7) shows three groups of quasi-implicative relationships among 17 of the 48 variables considered. First, recognise a polygon (variables 1a.2, 1a.9, 1a.4, 1a.11, 1a.13 and 1a.7; Figure 8a); second, transform a non-example of polygon into a polygon-explain and draw - (variables 1b.oci-e\&d, 1d.ci-e\&d and 1c.oc-e\&d) and recognise a polygon (1a.12) (Figure 8b); and third, identify the common attribute of a group of polygons, decide whether a figure belongs to this group and identify an attribute to draw a certain figure (variables 2.cv/cx-e\&d, 3.6/n6-e\&d, 4a.s/ns-e\&d, 4b.sym-bel, 5.tri-bel, 6a.tzo\&ntzo-d and 6.tzo-bel; Figure 8c). To better differentiate the groups, we coloured the variables of each dimension in the implicative graph: the variables of recognising polygons (Dimension 1) are presented in green; the variables for identifying the common attribute of a set of polygons (Dimension 2) are shown in blue; variables for determining whether a polygon belongs to a class (Dimension 3) are yellow; and variables for drawing polygons with conditions (Dimension 4) are illustrated in orange.

The relationships between the variables related to recognising examples of different figures as polygons (Figure 8a) indicated that students differentiated the three relevant attributes in the polygon's definition (closed figure, with straight and non-intersecting sides) from other non-relevant attributes, such as being an irregular or regular figure, being a concave or convex figure, and having a different number of sides. In addition, the relationships between the variables that consist in turning a non-example of a polygon into a polygon (Figure 8b) indicated a certain dependence on the number of attributes that had to be modified. That is, when students turn an open figure with intersecting sides and a curved side into a polygon, they are also able to do so when only two 
attributes need to be modified. Finally, the relationships between the variables of identifying a polygon class and determining examples of this class showed that if students identify symmetric polygons as a class (4a.s/ns-e\&d) then they can identify other particular inclusion relationships (Figure 8c), such as: considering the rhombus as a quadrilateral with an axis of symmetry (4b.sym-bel); considering the equilateral triangle as an example of isosceles triangles (5.tri-bel); and considering the square as an example of trapezoid (quadrilateral with at least two parallel sides) (6b.tzo-bel).

In the following sections we describe the ideas above that characterise the understanding of the polygon concept and the relationships between polygons.

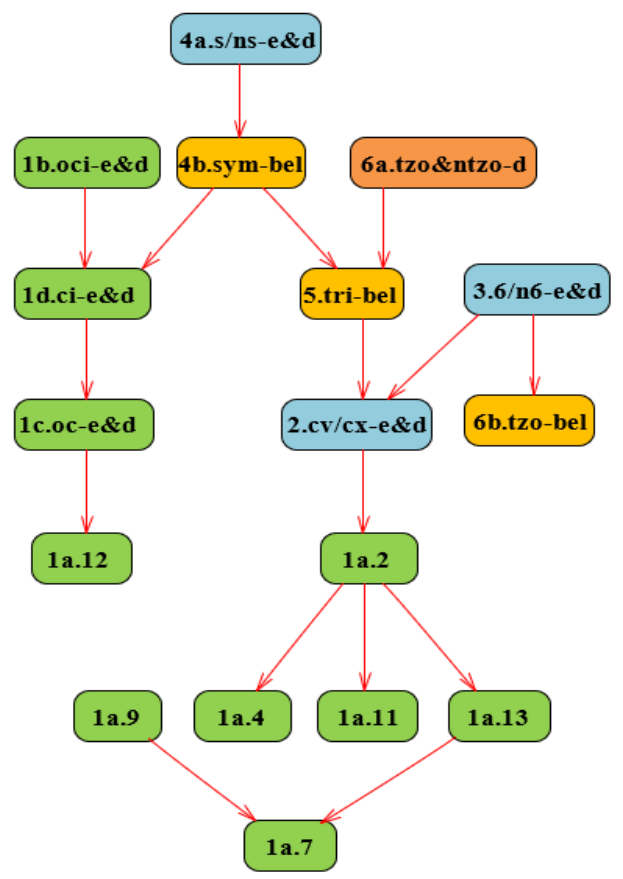

Figure 7. Implicative graph with 98\% significance showing the relationships between the attributes that define the concept of polygon in third year primary school students

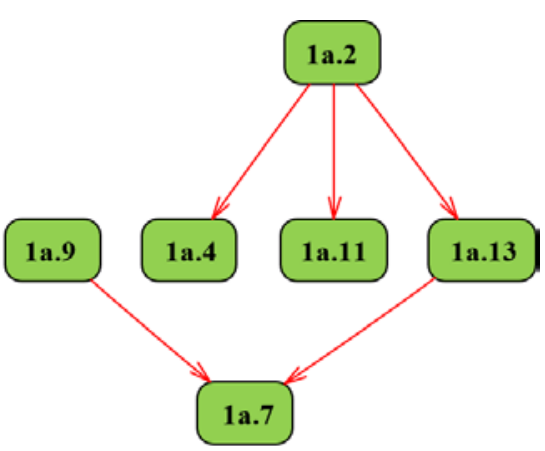

Figure 8a

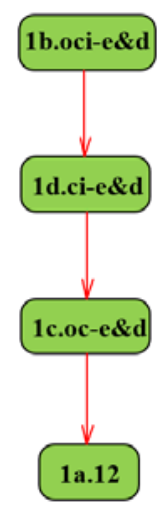

Figure $8 b$

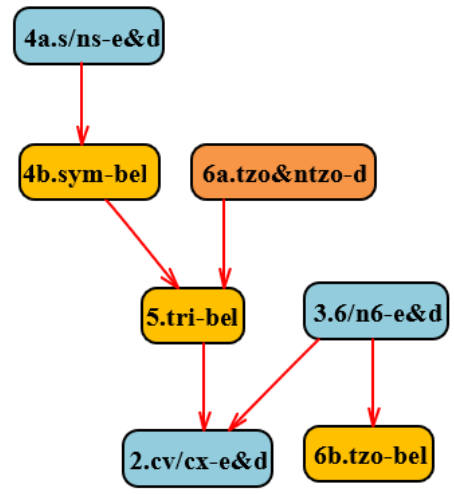

Figure 8c

Figure 8a. Dimension 1, Recognise polygons (Variables 1a.n)

Figure 8b. Dimension 1, Transform non-examples of polygons into polygons: draw and explain (Variables 1b.oci-e\&d, 1d.ci-e\&d, and 1c.oc-e\&d)

Figure 8c. Reason with attributes: Dimension 2, Identify a group's common attributes (Variables 2.cv/cx-e\&d, 3.6/n6-e\&d, and 4a.s/ns-e\&d), Dimension 3, establish relationships of inclusion (Variables 4b.sym-bel, 5.tri-bel and 6b.tzo-bel) and Dimension 4, identify parallelism to draw (Variable 6a.tzo\&ntzo-d) 


\section{Recognise polygons and transform a non-example of polygon into a polygon}

The quasi-implicative relationships between the variables that indicate the transformation of a non-example of a polygon into a polygon (Figure 8b) demonstrate the presence of the coordination between discursive apprehensions (assign mathematical properties to parts of a figure) and sequential apprehension (draw) considering the relevant attributes in the definition of polygon (closed 2D figure with straight and nonintersecting sides) with different semiotic registers (explain and draw). The quasi-implication 1b.oci-e\&d $\rightarrow$ 1d.ci-e\&d $\rightarrow$ 1c.oc-e\&d $\rightarrow$ 1a.12 (Figure 8b) indicates that explaining and transforming a non-example of a polygon into a polygon depends on the attributes considered. That is: when the figure is $2 \mathrm{D}$, open with intersecting sides and a curved side (1b.oci-e\&d); when the figure has intersecting sides, is 2D and with a curved side (1d.ci-e\&d); and when the figure is $2 \mathrm{D}$, open and with a curved side (1c.oc-e\&d). The dependence between the different attributes is given by the established quasi-implicative relationships. These quasi-implicative relationships indicate that when a student turns an open 2D figure with intersecting sides and a curved side into a polygon (1b.oci-e\&d) and explains the transformation of a 2D figure with curved and intersecting sides into a polygon (1d.ci-e\&d), then it is likely that the student can explain the transformation of an open 2D figure with curved sides into a polygon (1c.oc-e\&d).

This quasi-implicative relationship is illustrated by the answers of student G1S5 (student 5 in group 1). This student recognises the attributes that are not met in Figure "O" of item $1 \mathrm{~b}$ (1b.oci-e\&d) to be a polygon. This student explained that the figure should be closed and had to have straight and non-intersecting sides to be a polygon and drew the obtained polygon by modifying the attributes of the given figure (Figure 9a). In item 1d, (1d.ci-e\&d) the student explained that in Figure "Q”, the sides had to be put straight, that to be a polygon, they must not be intersecting and drew a polygon based on this explanation (Figure 9b). In addition, this student explained which attributes had to be modified in Figure "P" of item 1c (1c.oc-e\&d) to obtain a polygon. The student indicated that it should be closed, that the curved side would have to be turned into a straight side and drew the polygon to illustrate this transformation (Figure 9c).

\section{Item 1b}

Figure "O" is not a polygon. Explain in your own words what you would change to turn it into a polygon. Draw it.

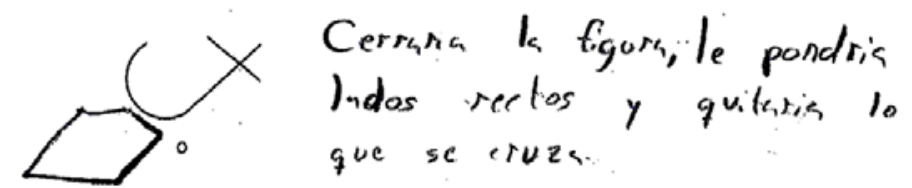

I would close the figure, I would put straight sides and remove those that are intersecting.

Figure 9a. Variable 1b.oci-e\&d. Answer of student G1S5 to item 1b. Transform an example of non-example of polygon into a polygon (attributes to be changed: open figure, curved side, intersecting sides)

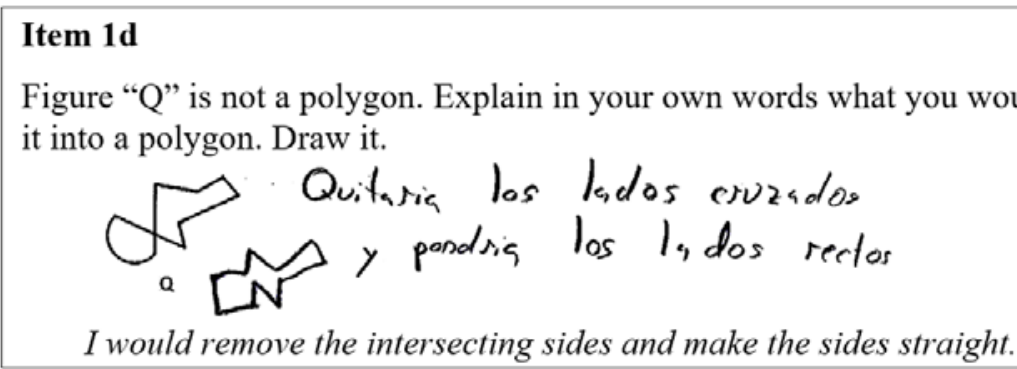

Figure 9b. Variable 1d.ci-e\&d. Answer of student G1S5 to item 1d. Transform an example of non-example of polygon into a polygon (attributes to be changed: intersecting sides, and curved side) 


\section{Item 1b}

Figure "P" is not a polygon. Explain in your own words what you would change to turn it into a polygon. Draw it.

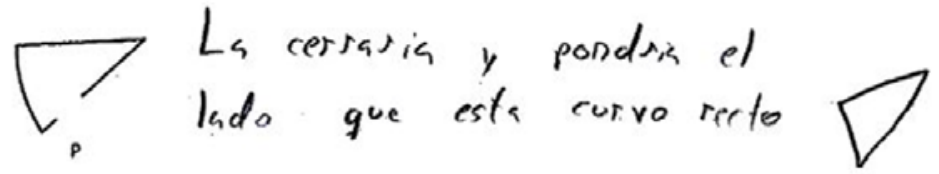

I would close it and turn the curved side into a straight side.

Figure 9c. Variable 1c.oc-e\&d. Answer of student G1S5 to item 1c. Transform an example of non-example of polygon into a polygon (attribute to be changed: open figure and curved side)

\section{Identifying a polygon class and determining the belonging to the class}

The implications between the variables "identify classes of polygons" and inclusion relationships (Figure 8c) demonstrate the role played by the different attributes under consideration. The implications show the hierarchy between:

- $\quad$ the identification of symmetry (variables 4a.s/ns-e\&d and 4b.sym-bel) to define a class;

- the inclusion relationship when an equilateral triangle is considered as an isosceles triangle (variable 5.tri-bel);

- the identification of the number of sides as the attribute defining a class (variable 3.6/n6-e\&d);

- the identification of concavity/convexity as the attribute defining a class (variable 2.cv/cx-e\&d); and

- recognising the square as a quadrilateral with two parallel sides (variable 6b.tzo-bel).

The quasi-implicative relationships generated (4a.s/ns-e\&d $\rightarrow$ bb.sym-bel $\rightarrow$ 5.tri-bel) indicate that if students identify the symmetry of the figures as the attribute that defines a class (variable 4a.s/ns-e\&d) and considers the rhombus as an example of the polygons with an axis of symmetry (variable 4b.sym-bel), then they are likely to consider the equilateral triangle as an example of an isosceles triangle (variable 5.tri-bel). In addition, the quasiimplication 5.tri-bel $\rightarrow$ 2.cv/cx-e\&d indicates that if students consider the equilateral triangle as an example of isosceles triangle then they are likely to identify concavity as an attribute that defines a class of polygons. In addition, the quasi-implication 6a.tzo\&ntzo-d $\rightarrow$ 5.tri-bel indicates that if students draw at least two quadrilaterals with two parallel sides and at least two quadrilaterals without parallel sides (variable 6a.tzo\&ntzod) when an oral description is provided, then it is likely that they recognise the equilateral triangle as an example of isosceles triangle (variable 5.tri-bel). Finally, if students are able to identify "having six sides" as an attribute that defines a class of polygons (3.6/n6-e\&d), then they are likely to identify concavity as an attribute that defines a class of polygons (2.cv/cx-e\&d) and can also identify the square as an example of quadrilateral with two parallel sides (6b.tzo-bel).

The answers of student G1S18 (student 18 of group 1) provide evidence of these quasi-implications (6a.tzo\&ntzo-d $\rightarrow$ 5.tri-bel $\rightarrow$ 2.cv/cx-e\&d). In this case, the student drew at least two quadrilaterals with two parallel sides when an oral description was provided, and two others without parallel sides (Variable 6a.tzo\&ntzo-d, Figure 10a) and explained that a triangle with three equal sides (equilateral) was an example of an isosceles triangle (a triangle with at least two equal sides) (Variable 5.tri-bel, Figure 10b). Thus, the student was able to identify concavity (being concave or convex) as an attribute that defines a class, and of drawing an example and a non-example of this group (variable 2.cv/cx-e\&d, Figure 10c).

\section{Item 6a}

A Drawing machine can draw:

Quadrilaterals (polygons with four sides) with two parallel sides

a) Draw three quadrilaterals that the machine can draw, and three quadrilaterals that the machine cannot draw

\begin{tabular}{c|c} 
CAN DO & CANNOT DO \\
Quadrilaterals with two parallel sides & \\
\hline
\end{tabular}




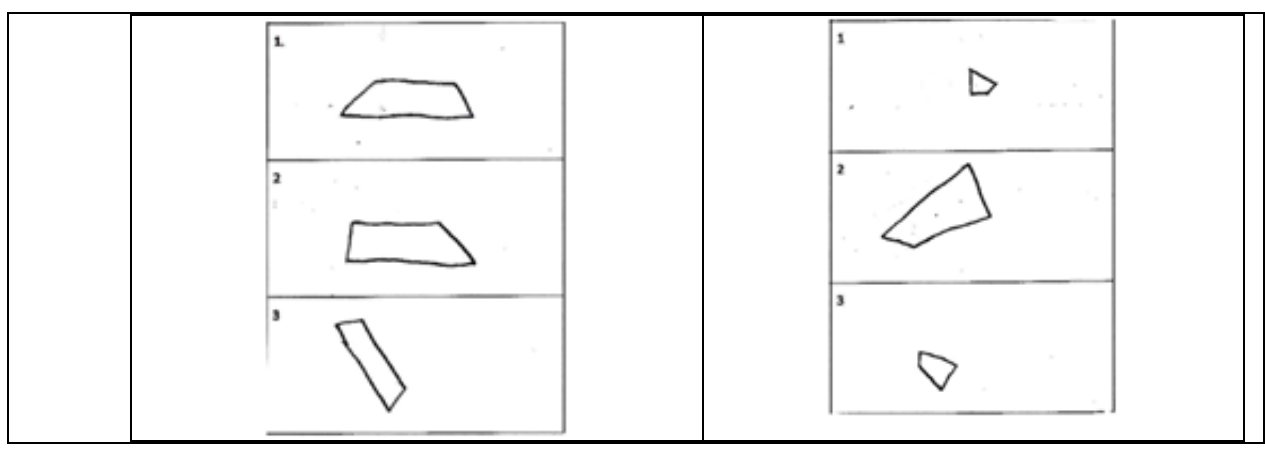

Figure 10a. Variable 6a.tzo\&ntzo-d. Answer of student G1S18 to item 6a. Draw three quadrilaterals with two parallel sides and three quadrilaterals without parallel sides

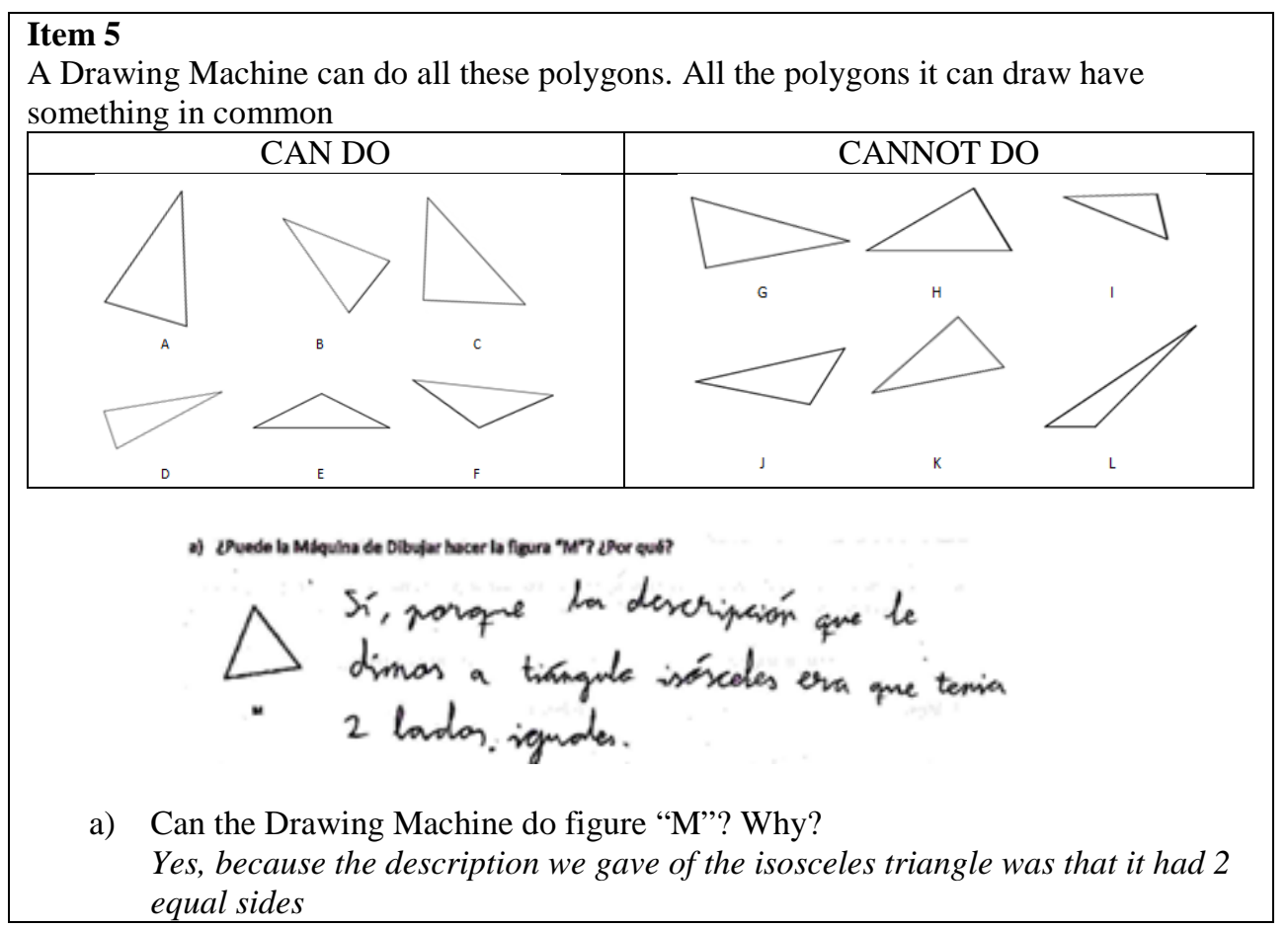

Figure 10b. Variable 5.tri-bel. Answer of student G1S18 to item 5. Explain why a triangle with three equal sides (equilateral) is an example of a triangle with at least two equal sides (isosceles)

\section{Item 2}

A Drawing Machine can do all these polygons. All the polygons it can draw have something in common

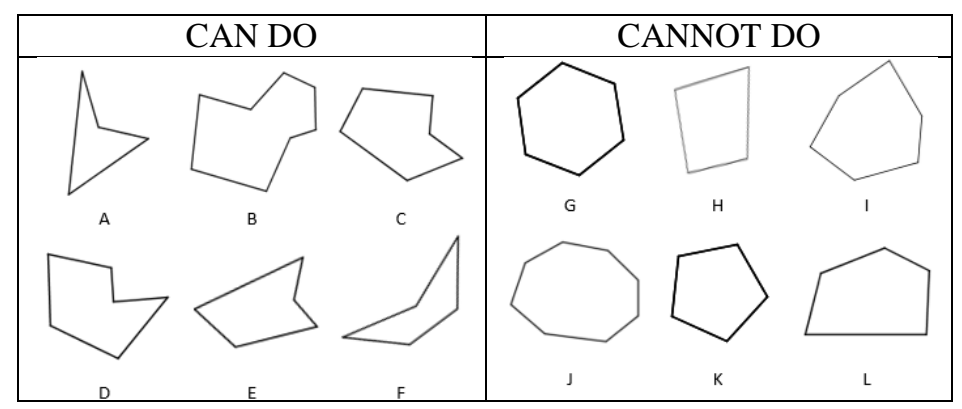

a) Draw different polygons that the Drawing machine can do and explain why 


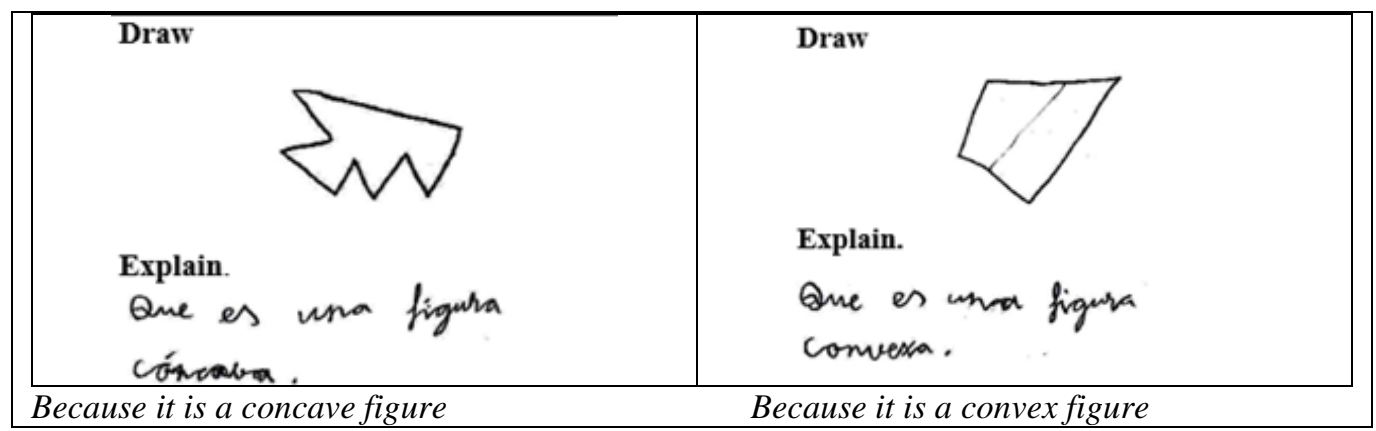

Figure 10c. Variable 2.cv/cx-e\&d. Answer of student G1S18 to Item 2. Identify and explain why the Drawing Machine can make concave polygons and cannot make convex polygons. Moreover, draw a figure that could belong to the class of concave figures, and an example that does not belong to this class.

\section{DISCUSSION}

The objective of this study was to characterise how third-grade primary school students understand the concept of polygon and how they consider a particular polygon as an example of a class. In other words, we sought to determine how students recognise the relevant attributes in the definition of polygon (attributes that a figure must have to be a polygon) and how they reason with non-relevant attributes in the definition (attributes that only some examples of polygons have such as: concavity/convexity, symmetry, or a specific number of sides). The dual process of recognising attributes in a figure and identifying a common attribute in two different figures relies on the ability to coordinate different apprehensions and on the capacity for dimensional deconstruction, which allows to identify sub-configurations with a mathematical meaning (Duval 2017). Two relevant findings emerged from our results. First, a progressive complexity underlies the understanding of the concept of polygon, which was evidenced by the relationship between recognising a figure as a polygon and transforming a nonexample of a polygon into a polygon. Second, understanding that a specific polygon is an instance of a class of polygons depends on the attribute that defines the class. That is, the fact of identifying a class of polygons is linked to taking non-relevant attributes into account (symmetry, concavity/convexity, number of sides, parallelism and length of the sides in the triangles). This fact indicates that the cognitive processes of identifying and determining a class of polygons are related to recognising a polygon's specific non-relevant attributes.

\section{Progressive complexity in understanding the concept of polygon}

Students' understanding of the concept of polygon rests on a progressive complexity, which in turn, is based on two abilities. The first is being able to differentiate examples and non-examples of polygons, recognising that a figure meets the relevant attribute included in the definition of a polygon (closed 2D figure delimited by straight, non-intersecting sides). The second is the ability to turn non-examples of polygons into polygons, to explain such a transformation and to draw the final polygon, relating the recognition of certain attributes and the mathematical properties that define the polygon concept (logical conditions governing the concept) (figural concept) (Fischbein 1993; Fujita and Jones 2007). The fact that an individual recognises examples and nonexamples and turns non-examples into examples is evidence of suitable interactions between concepts and images (Hershkowitz 1990). Our results indicate that students seem to some extent, to build the conceptual structure of the polygon concept depending on the different attributes that must be recognised or modified, thus revealing the underlying role of perceptual, operative and discursive apprehensions in understanding geometrical figures (Gagatsis et al. 2010; Karpuz and Atasoy 2019).

The quasi-implicative relationships show a hierarchy in the roles played by different attributes in students' ability to recognise a figure's attributes that must be modified in order to turn it into a polygon and when one, two or three attributes must be modified (Figure 6a). That is, in the way in which students recognise when "an instance is missing at least one critical attribute of the concept being considered" (Tsamir et al. 2008). This result broadens our knowledge about the way in which children aged 3 to 6 years recognise different types of figures as polygons (Clements et al. 1999), specifically regarding how primary students extend the recognition of nonexamples of polygons (Tsamir et al. 2008). This result indicates that dimensional deconstruction (Duval 2017) 
through the coordination of apprehensions depends on the attributes considered and the amount of attributes that must be taken into account at the same time. To perform a dimensional deconstruction, students must recognise and give a mathematical meaning to the straight sides, be a closed figure and has not cross sides in order to perform the discursive apprehension (Duval 1995). This coordination is visible in the quasi-implicative relationships generated between the variables related to turning a non-example of a polygon into a polygon since the implicative graph showed the variables that combine the discursive and non-discursive registers (1c.oc-e\&d instead of 1c.oc-e or 1c.oc-d) (Duval 2017). In the same way that pre-school students are able to name figure attributes depending on the type of figure (Clements et al. 1999), our results indicate that the way in which thirdgrade primary students transform a figure into a polygon depends on the attribute that must be modified.

\section{Identify belonging to a class}

Our results suggest that following a teaching experiment focused on recognising polygons and different attributes beyond those provided in the definition, third-grade primary school students can be relatively successful in determining polygon classes and considering specific inclusion relationships (when a figure is an example of a class). Furthermore, the findings indicate that the dimensional deconstruction underlying the categorisation process depends on the attribute used to classify (concavity/convexity, number of sides, symmetry, and parallelism). Two conclusions can be drawn from this.

First, the relationships relating to the identification of the different attributes that define a polygon class seem to indicate particular developments depending on the attributes. That is to say, the quasi-implicative relationships indicate that dimensional deconstruction depends on the attributes. The quasi-implication among the polygons' attributes—such as number of sides, concavity and symmetry-illustrates specific aspects of the understanding of relationships between polygons. This fact sheds light on the fact that third-grade primary school students begin to determine that a figure belongs to a class based on the mathematical meaning given to some subconfiguration of the figures through the apprehensions linked to dimensional deconstruction. This way of reasoning underscores that primary school students may be able to identify some attributes of geometric figures and give them mathematical meaning (being symmetrical, having a certain number of sides and concavity/convexity), examining their parts and establishing differences and similarities between their parts. This mode of reasoning has been reported in secondary school students incorporating the use of properties to infer polygon characteristics (Fujita 2012) and to characterise the ability to represent and interpret 3D geometric relations (Pittalis and Christou 2013). Our findings provide evidence of this cognitive ability in primary school students to understand an instance of polygon as an example of a class.

Second, the results demonstrate the relevance of recognising symmetry and parallelism: if students recognise symmetry and draw quadrilaterals with or without parallel sides, they can then identify the number of sides and concavity/convexity as the characteristics of a class. That is, if the student identifies symmetry as the class that defines the set of polygons and draws examples and non-examples of quadrilaterals with two parallel sides, he or she might be able to identify concavity as the attribute that defines the class. However, if a student identifies concavity as the attribute that defines the class, this does not imply that he or she will identify symmetry as the class that defines the set of polygons or will draw examples or non-examples of quadrilaterals with two parallel sides. These results suggest the relevant role of symmetry and parallelism in making logical inferences that allow organising figures according to their characteristics, and therefore as a characteristic of primary students' categorical thinking (Clements et al. 1999).

\section{CONCLUSIONS}

Globally, our results show that students solved items simultaneously at multiple levels: this suggests that the understanding of the concept of polygon involves a mixture of visual and analytical processes (Fischbein 1993; Hershkowitz 1989) depending on the attributes taken into consideration. In addition, our findings indicate a gradual development of primary students' categorical thinking regarding polygon classes.

These results shed light on the complexity of the reasoning linked to specific attributes, determining the way in which students begin to understand the idea of belonging to a class. Our results support the arguments that challenge the hypothesis according to which the development of geometric thinking is hierarchical (Clements 
and Battista 1992). In this sense, the quasi-implicative diagrams can be understood as a visual representation of the meaning of the "hierarchical" development in the sense of that the students' answers that do not fulfil the implicative relationship is relatively small. The theoretical perspective of dimensional deconstruction and cognitive apprehensions, together the implicative analysis, provided us with information on the role of polygon attributes to identify examples and non-examples of a class and how we can understand the nature of the development of geometrical thinking. This study shows that recognising a polygon as an example of a class depended on the attribute that defines the class (for example, symmetry, parallelism and concavity/convexity). Furthermore, our findings point to the need to consider the relationships between cognitive processes - such as identify a figure as an instance of a class — and geometrical content—such as parallelism, symmetry, concavity — when approaching the development of geometrical thinking. This involves considering several dimensions when describing the understanding of geometrical figures (Gagatsis et al. 2010).

Three important implications for teaching can be drawn from the results of this study. First, the need to define the relationship between perceptual, operative, discursive and sequential apprehensions as a teaching objective, explicitly considering the attributes' variability in the items provided. That is, teaching should focus on recognising examples and non-examples, and transforming non-examples into examples of the geometric concepts (explain and draw) to stimulate the understanding of the polygon concept. Second, teaching should focus on dimensional deconstruction and the coordination of apprehensions to analyse figures, in order to strengthen students' ability to differentiate figures, taking various attributes into account, as well as their ability to assign mathematical meaning. Finally, teaching should also focus on enhancing primary students' recognition of figures as examples of classes of figures. These instructional principles would help to broaden primary students' usual range of examples and non-examples, enabling them to generate analytical judgments based on figure attributes.

\section{References}

Battista, M. T. (2007). The development of geometric and spatial thinking. In F. K. Lester (Ed.), Second handbook of research on mathematics teaching and learning (pp. 843-908). Reston, VA: NCTM-IAP.

Battista, M. (2012). Cognition-based Assessment \& Teaching of Geometric Shapes. Building on students' reasoning. Portsmouth, $\mathrm{NH}$ : Heinemann.

Bernabeu, M., \& Llinares, S. (2017). How do six to nine years-old children understand geometrical shapes. Educación Matemática, 29(2), 9-35, https://doi.org/10.24844/EM2902.01

Bernabeu, M., Moreno, M., \& Llinares, S. (2018). Primary School Children's (9 years-old) understanding of quadrilaterals. In E. Bergqvist, M. Österholm, C. Granberg, \& L. Sumpter (Eds.), Proceedings of the 42nd Conference of the International Group for the Psychology of Mathematics Education (Vol. 2, pp. 155-162). Umeå, Sweden: PME.

Clements, D. H., \& Battista, M. T. (1992). Geometry and spatial reasoning. In D. A. Grouws (Ed.), Handbook of research on mathematics teaching and learning (pp. 420-464). New York, NY: MacMillan.

Clements, D., Swaminathan, S., Hannibal, M., \& Sarama, J. (1999). Young children’s concepts of shape. Journal for Research in Mathematics Education, 30(2), 192-212. https://doi.org/10.2307/749610

De Villiers, M. (1994). The role and function of a hierarchical classification of quadrilaterals. For the Learning of Mathematics, 14(1), 11-18.

Duval, R. (1995). Geometrical pictures: Kinds of representation and specific processings. In R. Sutherland \& J. Mason (Eds.), Exploiting mental imagery with computers in mathematics education (pp. 142-157). Berlin, DE: Springer.

Duval, R. (1999). Representation, Vision and Visualization: Cognitive Functions in Mathematical Thinking. In F. Hitt \& M. Santos (Eds.), Proceedings of the 21st Annual Meeting North American Chapter of the International Group of PME (pp. 3-26). Columbus, OH: ERIC/CSMEE.

Duval, R. (2017). Understanding the mathematical way of thinking - The registers of semiotic representations. Cham, BY: Springer. https://doi.org/10.1007/978-3-319-56910-9

Fischbein, E. (1993). The theory of figural concepts. Educational Studies in Mathematics, 24, 139-162. 
Fujita, T. (2012). Learners' level of understanding of the inclusion relations of quadrilaterals and prototype phenomenon. The Journal of Mathematical Behavior, 31(1), 60-72.

Fujita, T., \& Jones, K. (2007). Learners' understanding of the definitions and hierarchical classification of quadrilaterals: Towards a theoretical framing. Research in Mathematics Education, 9(1, 2), 3-20.

Fujita, T., Doney, J., \& Wegerif, R. (2019). Students' collaborative decision-making processes in defining and classifying quadrilaterals: a semiotic/dialogic approach. Educational Studies in Mathematics, 101, 341-356. https://doi.org/10.1007/s10649-019-09892-9

Gagatsis, A., Sriraman, B., Elia, I., \& Modestou, M. (2006). Exploring young children’s geometrical strategies. Nordic Studies in Mathematics Education, 11(2), 23-50.

Gagatsis, A.; Monoyiou, A.; Delilyianni, E.; Elia, I.; Michael, P.; Kalogirou, P., Panaoura, A.\&, Philippou, A. (2010). One way of assessing the understanding of a geometrical figure. Acta Didactica Universitatis Comenianae Mathematics, 10, 35-50.

Gras, R., \& Kuntz, P. (2008). An overview of the Statistical Implicative Analysis (SIA) development. In Gras R., Suzuki E., Guillet F. \& Spagnolo F. (Eds.), Statistical implicative analysis (pp. 11-40). Heidelberg, BW: Springer.

Gras, R., Suzuki, E., Guillet, F., \& Spagnolo, F. (2008). Statistical Implicative Analysis. Theory and Applications. London, UK: Springer.

Halat, E., \& Yesil-Dagli, U. (2016). Preschool students' Understanding of a Geometric Shape, the Square. BOLEMA, 30(55), 830-848. https://doi.org/10.1590/1980-4415v30n55a25

Hershkowitz, R. (1989). Visualization in geometry: Two sides of the coin. Focus on Learning Problems in Mathematics, 11(1), 61-76.

Hershkowitz, R. (1990). Psychological aspects of learning geometry. In P. Nesher \& J. Kilpatrick (Eds.), Mathematics and cognition (pp. 70-95). Cambridge, UK: Cambridge University Press.

Karpuz, Y., \& Atasoy, E. (2019) Investigation of $9^{\text {th }}$ Grade Students' Geometrical Figure Apprehension. European Journal of Educational Research, 8(1), 285-300.

Kaur, H. (2015). Two aspects of young children's thinking about different types of dynamic triangles: prototypicality and inclusion. ZDM. Mathematics Education, 47, 407-420. https://doi.org/10.1007/s11858$\underline{014-0658-\mathrm{Z}}$

Kozakli-Ulger, T., \& Tapan-Broutin, M.S. (2017). Pre-service Mathematics Teachers' Understanding of Quadrilaterals and the internal relationships between quadrilaterals: The case of parallelograms. European Journal of Educational Research, 6(3), 331-345.

Mesquita, A. L. (1998). On conceptual obstacles linked with external representations in Geometry. Journal of Mathematical Behavior, 17(2), 183-195.

Michael, S., Elia, I., Gagatsis, A., Theoklitou, A., \& Savva, A. (2006). Levels of understanding of patterns in multiple representations. In J. Novotna, H. Moraova, M. Kratka \& N. Stehlikova (Eds.), Proceedings of the 30th Conference of the International Group for the Psychology of Mathematics Education (Vol. 4, pp. 161168). Prague, Czech Republic: PME.

Monaghan, F. (2000). What difference does it make? Children views of the difference between some quadrilaterals. Educational Studies in Mathematics, 42(2), 179-196.

Moreno, M., Bernabeu, M., Gagatsis, A., Llinares, S., \& Panaoura, R. (2019). Changes in third-grade students' understanding of polygons. In M. Graven, H. Venkat, A. Essien \& P. Vale (Eds.), Proceedings of the 43rd Conference of the International Group for the Psychology of Mathematics Education (Vol. 3, pp 97-104). Pretoria, South Africa: PME.

Satlow, E., \& Newcombe, N. (1998). When is a triangle not a triangle? Young children's developing concepts of geometric shape. Cognitive Development, 13(4), 547-559. https://doi.org/10.1016/S0885-2014(98)90006-5

Sinclair, N., Bartolini Bussi, M. G., de Villiers, M., Jones, K., Kortenkamp, U., Leung, A., \& Owens, K. (2016). Recent research on geometry education: An ICME-13 survey team report. ZDM. Mathematics Education, 48(5), 691-719.

Sinclair, N., Cirillo, M., \& de Villiers, M. (2017). The learning and teaching geometry. In J. Cai (Ed.), Compendium for research in mathematics education (pp. 457-489). Reston, VA: NCTM. 
Trigueros, M., \& Escandón, C. (2008). Los conceptos relevantes en el aprendizaje de la graficación. Un análisis a través de la estadística implicativa. Revista Mexicana de Investigación Educativa, 13(36), 59-85.

Tsamir, P., Tirosh, D., \& Levenson, E. (2008). Intuitive nonexamples: the case of triangles. Educational Studies in Mathematics, 69, 81-95. https://doi.org/10.1007/s10649-008-9133-5

Tsamir, P., Tirosh. D., Levenson, E., Barbakai, R., \& Tabach, M. (2015). Early-years teachers’ concept images and concept definitions: triangles, circles, and cylinders. ZDM. Mathematics Education, 47, 497-509.

Walcott, C., Mohr, D., \& Kastberg, S. (2009). Making sense of shape: An Analysis of children's written responses. Journal of Mathematical Behavior, 28, 30-40. https://doi.org/10.1016/j.jmathb.2009.04.001

Wang, S., \& Kinzel, M. (2014). How do they know it is a parallelogram? Analysing geometric discourse at van Hiele Level 3. Research in Mathematics Education, 16(3), 288-305. https://doi.org/10.1080/14794802.2014.933711

Yesil-Dagli, U., \& Halat, E. (2016). Young children’s conceptual understanding of triangle. Eurasia Journal of Mathematics, Science \& Technology Education, 12(2), 189-202. https://doi.org/10.12973/eurasia.2016.1398a 